\title{
Breast dynamic contrast enhanced MRI: fibrocystic changes presenting as a non-mass enhancement mimicking malignancy
}

\author{
Zorica C. Milosevic ${ }^{1,2}$, Mirjan M. Nadrljanski ${ }^{1,2}$, Zorka M. Milovanovic ${ }^{3}$, Nina Z. Gusic ${ }^{4}$, \\ Slavko S. Vucicevic ${ }^{1}$, Olga S. Radulovic ${ }^{5}$ \\ ${ }^{1}$ Clinic for Radiation Oncology and Radiology, Institute of Oncology and Radiology of Serbia, Belgrade, Serbia \\ ${ }^{2}$ Faculty of Medicine, University of Belgrade, Belgrade, Serbia \\ ${ }^{3}$ Department for Pathology, Institute of Oncology and Radiology of Serbia, Belgrade, Serbia \\ ${ }^{4}$ Primary Health Center Zvezdara, Belgrade, Serbia \\ ${ }^{5}$ Institute for Biological Research 'Sinisa Stankovic', Belgrade, Serbia
}

Radiol Oncol 2017; 51(2): 130-136.

Received 18 October 2016

Accepted 20 February 2017

Correspondence to: Zorica Miloševic, M.D., Clinic for Radiation Oncology and Radiology, Institute of Oncology and Radiology of Serbia, Pasterova 14, 11000 Belgrade, Serbia and Faculty of Medicine, University of Belgrade, Dr Subotića 8, 11000 Belgrade, Serbia.

Phone: +381 11206 7214; Fax: +38111268 53 00; E-mail: pipa011@ptt.rs

Disclosure: No potential conflicts of interest were disclosed.

Background. We aimed to analyse the morphokinetic features of breast fibrocystic changes (nonproliferative lesions, proliferative lesions without atypia and proliferative lesions with atypia) presenting as a non-mass enhancement (NME) in dynamic contrast-enhanced magnetic resonance imaging (DCE-MRI) examination.

Patients and methods. Forty-six patients with histologically proven fibrocystic changes (FCCS) were retrospectively reviewed, according to Breast Imaging Reporting and Data System (BI-RADS) lexicon. Prior to DCE-MRI examination, a unilateral breast lesion suspicious of malignancy was detected clinically, on mammography or breast ultrasonography.

Results. The predominant features of FCCs presenting as NME in DCE-MRI examination were: unilateral regional or diffuse distribution (in 35 patients or $76.1 \%$ ), heterogeneous or clumped internal pattern of enhancement (in 36 patients or 78.3\%), plateau time-intensity curve (in 25 patients or 54.3\%), moderate or fast wash-in (in 31 patients or $67.4 \%$ ). Nonproliferative lesions were found in 11 patients $(24 \%)$, proliferative lesions without atypia in 29 patients $(63 \%)$ and lesions with atypia in six patients (13\%), without statistically significant difference of morphokinetic features, except of the association of clustered microcysts with proliferative dysplasia without atypia.

Conclusions. FCCs presenting as NME in DCE-MRI examination have several morphokinetic features suspicious of malignancy, therefore requiring biopsy (BI-RADS 4). Nonproliferative lesions, proliferative lesions without atypia and proliferative lesions with atypia predominantly share the same predefined DCE-MRI morphokinetic features.

Key words: breast; magnetic resonance imaging; fibrocystic changes

\section{Introduction}

Fibrocystic changes (FCCs) are the most frequent benign conditions of breast, diagnosed in $50 \%$ of women examined clinically and in $90 \%$ of women in histopathological studies. These benign disorders have two important implications from the point of view of breast cancer diagnosis and man- agement. First, FCCs can mimic breast cancer on clinical examination, mammography and breast ultrasonography, leading to unnecessary breast biopsies and patient anxiety. Second, some types of FCCs represent a risk factor for the subsequent development of breast cancer. ${ }^{1}$ Based on a classification system of FCCs proposed by Dupont and Page and according to other studies, women with 
histologically confirmed nonproliferative lesions have no increased breast cancer risk. On the contrary, women whose breast biopsies show proliferative lesions with or without atypia are at risk of developing cancer, with relative risk ranging from $3.9-13.0$ and $1.3-1.9$ respectively..$^{2-4}$

Combining morphological and enhancement kinetics features of the breast lesions, dynamic contrast-enhanced MRI (DCE-MRI) shows the highest sensitivity of all imaging methods in detecting breast diseases, up to $100 \%$. Nevertheless, the specificity in the differentiation between benign and malignant lesions is lower, up to $75 \% .^{5}$ The major cause of false positive findings in DCE-MRI examination and consecutive unnecessary biopsies are the lesions with non-mass enhancement (NME). NME refers to the lesion that is seen only on postcontrast DCE-MRI sequences and does not have space-occupying effect. The enhancement pattern of NME is distinct from normal surrounding breast parenchyma and may contain interspersed fat. On the contrary, a mass enhancement is a three-dimensional space-occupying lesion. ${ }^{6,7}$ The causes of NME include FCCs, inflammatory benign lesions, in situ ductal carcinoma (DCIS), invasive lobular carcinoma and some cases of oestrogen receptornegative invasive ductal carcinoma. The mass enhancement is usually confined to malignant or benign tumors. ${ }^{8-11}$

Our study of pathologically confirmed FCCs presenting as NME in DCE-MRI examination has two goals: 1) to analyse morphological and enhancement kinetics features of FCCs, 2) to compare these features between nonproliferative lesions, proliferative lesions without atypia and proliferative lesions with atypia.

\section{Patients and methods}

\section{Patients}

In the period of two years (January 2010 to January 2012) a total of 947 patients were examined by two radiologists (MZC and NMM) using the standardized breast DCE-MRI full diagnostic protocol. From this group, 46 patients with FCCs presenting as NME were selected and retrospectively reviewed. The study was performed in accordance with the Declaration of Helsinki and was approved by the Institutional Review Board Committee (No. 4502-01/2011). All patients gave written informed consent to participate in DCE-MRI examination. The age of the patients was $50.78 \pm 8.99$ years. In all premenopausal women DCE-MRI was performed in the second and third week of the menstrual cycles. None of the patients had previous breast biopsy, breast surgery or current hormone replacement therapy. All patients initially had unilateral breast lesion suspicious of malignancy either on clinical examination, mammography or ultrasonography. The lesions were presented as calcifications or asymmetric tissue on mammography and hypoechoic non-mass lesions on ultrasonography (BIRADS 4a to BI-RADS 4c categories according to the ACR BI-RADS lexicon). ${ }^{6,12}$ The indication for breast DCE-MRI prior to biopsy was to evaluate the local extent of the lesion in the dense breast on mammography (32 patients) or suspected multifocal lesions on mammography and/or ultrasonography (14 patients). ${ }^{13}$ After the pathological confirmation of FCCs, the patients were examined biannually by physical examination and mammography. During the predefined follow-up period, no ipsilateral or contralateral breast cancer was detected.

\section{Methods}

The DCE-MRI examinations were performed with a 1.5 Tesla MRI unit (Magnetom Avanto, Siemens Medical Solutions, Erlangen, Germany) with dedicated bilateral breast coil and the patient in the prone position. The standard protocol was used for the axial-plane images with the slice thickness of $2 \mathrm{~mm}$ (Table 1). ${ }^{14}$ The contrast medium was gadopentetate dimeglumine (Magnevist, Bayer Schering Pharma, Berlin, Germany) applied as the bolus injection of $0.1 \mathrm{mmol} / \mathrm{kg}$ body weight injected with the automatic injector (Mississippi, Ulrich Medical,

TABLE 1. Standard dynamic contrast-enhanced magnetic resonance imaging (DCE-MRI) protocol for axial-plane images for T1-weighted FLASH 3D precontrast and five postcontrast series

\begin{tabular}{lcccc}
\hline & $\begin{array}{c}\text { T2- } \\
\text { weighted }\end{array}$ & $\begin{array}{c}\text { T2- } \\
\text { weighted }\end{array}$ & $\begin{array}{c}\text { T1- } \\
\text { weighted }\end{array}$ & $\begin{array}{c}\text { T1- } \\
\text { weighted }\end{array}$ \\
\hline $\begin{array}{l}\text { MRI sequence/ } \\
\text { parameters }\end{array}$ & TIRM & TSE & TSE & FLASH 3D \\
$\begin{array}{l}\text { Echo time (ms) } \\
\begin{array}{l}\text { Repetition time } \\
\text { (ms) }\end{array}\end{array}$ & 60 & 70 & 12 & 4.8 \\
$\begin{array}{l}\text { Inversion time (ms) } \\
\text { Flip angle ( }{ }^{\circ} \text { ) }\end{array}$ & 180 & 5900 & 910 & 9.1 \\
$\begin{array}{l}\text { Field of view } \\
\text { (mm } \times \text { mm) }\end{array}$ & 150 & 180 & 90 & 25 \\
Image matrix & $340 \times 340$ & $340 \times 340$ & $340 \times 340$ & $340 \times 340$ \\
\hline
\end{tabular}

$3 \mathrm{D}=$ three-dimensional; $\mathrm{DCE}-\mathrm{MRI}=$ dynamic contrast-enhanced magnetic resonance imaging; FLASH = fast low-angle shot pulse sequence; TIRM = turbo inversion recovery magnitude; TSE = turbo spin-echo 




FIGURE 1. DCE-MRI findings of proliferative fibrocystic changes. Axial Tl-weighted images, precontrast image (A) and contrast-enhanced image 1 min $23 \mathrm{~s}$ after administration of gadolinium (B) with corresponding subtraction image (C), show the segmental, non-mass enhancement (long arrows), measured $2.5 \times 1 \times 2.5 \mathrm{~cm}$, with clumped internal pattern of enhancement. Time-intensity curve is plateau (type 2) with fast wash-in (D). Turbo inversion recovery magnitude (TIRM) sequence (E) show clustered microcysts (short arrows).

Ulm, Germany) at the rate of $2 \mathrm{~mL} / \mathrm{s}$, followed by the flush of $20 \mathrm{~mL}$ saline. Contrast-enhanced dynamic sequences were acquired five times every $1 \mathrm{~min} 23$ $\mathrm{s}$. The postprocessing methods, including a creation of time intensity curves (TICs), i.e. enhancement (\%) against time (s), were performed on the workstation Leonardo, using the image processing software Syngo (Syngo, Siemens Medical Solutions).

Two experienced radiologists in breast MRI, ZCM, 20 yr. and MMN, 7 yr., reviewed the DCEMRI examinations. Based on the fifth edition of the BI-RADS lexicon, published in 2013, the following morphokinetic features of NME were analysed: distribution of enhancement (focal, linear, segmental, regional, multiple regions, diffuse), internal enhancement pattern (homogeneous, heterogeneous, clumped, clustered ring enhancement, stippled), type of TICs (persistent curve or type 1, plateau curve or type 2, wash out curve or type 3), washin i.e. the enhancement rate $90 \mathrm{~s}$ after intravenous contrast application (slow, medium, rapid), and signal intensity on T2-weighted (T2W) images. ${ }^{6}$

A surgical biopsy was performed in all patients. Among them, 21 patients with nonpalable lesions, prior to the biopsy underwent a radioguided occult lesion localization (ROLL) procedure with Technetium-99m, under stereotactic or ultrasonographic guidance. ${ }^{15}$ Haematoxylin and eosin stained slides of formalin-fixed and paraffin-embedded tissue blocks were assessed by a pathologist, experienced in breast pathology. According to the classification system of Dupont and Page, the lesions were classified as nonproliferative lesions, proliferative lesions without atypia, and proliferative lesions with atypia (atypical ductal and lobular hyperplasia). ${ }^{2}$

\section{Statistical analysis}

Following DCE-MRI features of NME were analysed: distribution of postcontrast enhancement, internal enhancement pattern, type of TICs, washin, and signal intensity on $\mathrm{T} 2 \mathrm{~W}$ images. These distinctive DCE-MRI features of NME were compared for three groups of FCCs: nonproliferative lesions, proliferative lesions without atypia, and proliferative lesions with atypia. Frequencies were used to describe the distribution of categorical variables. The difference between investigated variables in three groups of FCCs was analysed using $\chi^{2}$ test. $P$ values less than 0.05 were considered statistically significant. SPSS for Windows, Statistics version 16.0. (SPSS Inc., Chicago, USA) was used to perform statistical analyses.

\section{Results}

The lesions were detected clinically in 25 patients $(54.3 \%)$, on mammography in 43 patients $(93.5 \%)$ and by breast ultrasonography in 30 patients $(65.2 \%)$.

Out of 46 biopsies, nonproliferative lesions were found in 11 patients (24\%), proliferative lesions without atypia in 29 patients (63\%) and proliferative lesions with atypia in six patients (13\%). On DCE-MRI examination, all cases were presented as unilateral non-mass enhancement of BI-RADS 4 category (Figure 1).

Forty-three lesions (93.5\%) were equal to or larger than $1 \mathrm{~cm}$ in size and three lesions were smaller than $1 \mathrm{~cm}$ and larger than $0.5 \mathrm{~cm}$ in size (6.5\%). The distribution of the postcontrast enhancement was segmental in 11 patients $(23.9 \%)$ and regional or diffuse in 35 patients $(76.1 \%)$. The other types of distribution (focal, linear enhancement and multiple regions) were not found. The internal enhancement pattern was homogeneous in two patients (4.3\%), heterogeneous and clumped in 36 patients $(78.3 \%)$, and stippled in eight patients $(17.4 \%)$. Clustered ring enhancement was not detected. The types of time-intensity curves were persistent (type 1 ) in 17 patients (37.0\%), plateau (type 2 ) in 25 patients (54.3\%), and wash out (type 3 ) in four patients $(8.7 \%)$. The initial postcontrast signal in- 
TABLE 2. Nonproliferative lesion, proliferative lesions without atypia and proliferative lesions with atypia: dynamic contrastenhanced magnetic resonance imaging (DCE-MRI) morphological and enhancement kinetics features, based on BI-RADS lexicon

\begin{tabular}{|c|c|c|c|c|c|}
\hline \multirow{2}{*}{$\begin{array}{l}\begin{array}{l}\text { Statistical } \\
\text { difference }\end{array} \\
p=0.454\end{array}$} & \multirow{2}{*}{$\begin{array}{c}\begin{array}{c}\text { Proliferative lesions } \\
\text { without atypia }(\mathrm{N}=6)\end{array} \\
1\end{array}$} & \multirow{2}{*}{$\begin{array}{c}\text { Proliferative lesions } \\
\text { with atypia }(\mathrm{N}=29)\end{array}$} & \multirow{2}{*}{$\begin{array}{c}\text { Non proliferative } \\
\text { lesions }(\mathrm{N}=11)\end{array}$} & \multicolumn{2}{|c|}{$\begin{array}{l}\text { The DCE-MRI features of fibrocystic } \\
\text { changes }\end{array}$} \\
\hline & & & & $0.5<d<1 \mathrm{~cm}$ & Size \\
\hline & 5 & 28 & 10 & $\mathrm{~d} \geq 1 \mathrm{~cm}$ & \\
\hline \multirow[t]{2}{*}{$p=0.168$} & 3 & 7 & 1 & Segmental & NME distribution \\
\hline & 3 & 22 & 10 & Regional or diffuse & \\
\hline \multirow[t]{3}{*}{$p=0.722$} & 0 & 2 & 0 & Homogeneous & $\begin{array}{l}\text { NME internal } \\
\text { enhancement }\end{array}$ \\
\hline & 1 & 4 & 3 & Stippled & \\
\hline & 5 & 23 & 8 & $\begin{array}{l}\text { Heterogeneous or } \\
\text { clumped }\end{array}$ & \\
\hline \multirow[t]{3}{*}{$p=0.097$} & 3 & 8 & 6 & Persistent & TIC \\
\hline & 3 & 19 & 3 & Plateau & \\
\hline & 0 & 2 & 2 & Wash out & \\
\hline \multirow[t]{3}{*}{$p=0.752$} & 2 & 8 & 5 & Slow & Wash-in \\
\hline & 1 & 10 & 3 & Moderate & \\
\hline & 3 & 11 & 3 & Fast & \\
\hline \multirow[t]{2}{*}{$p=0.014$} & 1 & 18 & 2 & Present & $\begin{array}{l}\text { Microcysts (T2W } \\
\text { images) }\end{array}$ \\
\hline & 5 & 11 & 9 & Absent & \\
\hline
\end{tabular}

$\mathrm{d}=$ longest diameter; $\mathrm{DCE}-\mathrm{MRI}=$ dynamic contrast-enhanced magnetic resonance imaging; $\mathrm{NME}=$ non-mass enhancement; $\mathrm{TIC}=$ time-intensity curve $\mathrm{T} 2 \mathrm{~W}=\mathrm{T} 2$-weighted

tensity enhancement (enhancement rate, wash-in) was slow in 15 patients (32.6\%), moderate in 14 patients $(30.4 \%)$, and fast in 17 patients $(37.0 \%)$. On T2W images FCCs were associated with clustered microcysts in 25 cases (54.3\%).

As shown in Table 2, the features of nonproliferative lesions, proliferative lesions without atypia and proliferative lesions with atypia on DCE-MRI examination did not show statistically significant difference in the term of the size $(p=0.454)$, the distribution of postcontrast enhancement $(p=0.168)$, the internal enhancement pattern $(p=0.722)$, the types of time-intensity curves $(p=0.097)$ and the initial postcontrast signal intensity enhancement $(p$ $=0.752$ ). Presence of microcysts on T2W images in these three groups of FCCs was statistically significant compared to the lack of the feature $(p=0.014)$.

\section{Discussion}

Numerous studies have demonstrated the clinical importance of FCCs, related to the high prevalence of the condition, the considerable impact on quality of life and the increased breast cancer risk for women with proliferative lesions..$^{2-4,16-18}$
Nevertheless, only few studies analysed features of FCCs on DCE-MRI, based on the limited number of cases. Chen et al. reported two studies with 31 patients and 11 patients, analysing FCCs in DCEMRI examination and MR spectroscopy for choline detection. ${ }^{19,20}$ Van den Bosch et al. and Kiyak et al. reported morphological and kinetic features of FCCs on DCE-MRI in the group of 14 patients and 27 patients, respectively. ${ }^{21,22}$

Our study included 46 symptomatic patients with pathologically confirmed FCCs presenting as NME, mimicking malignancy in DCE-MRI examination. Chen et al. reported that FCCs on DCEMRI had characteristics of NME in 39\% of cases, mass enhancement in $35 \%$ of cases, while $26 \%$ of cases were nonenhancing lesions. ${ }^{19}$ According to the published data, breast DCE-MRI has the potential to distinguish benign from malignant mass lesions effectively. Nevertheless, DCE-MRI is inferior in discriminating benign from malignant NME lesions. ${ }^{23}$ Hence, a meticulous analysis of the multiple, standardized parameters from BI-RADS lexicon is crucial to achieve a higher diagnostic performance of DCE-MRI in the case of FCCs presenting as NME, including the distribution of enhancement, the internal enhancement pattern, the 
type of TICs, the wash-in, and the signal intensity on $\mathrm{T} 2 \mathrm{~W}$ images.

In our study the postcontrast enhancement of FCCs was unilateral in all patients. The most frequent types of the distribution of NME were regional - involving more than $25 \%$ of a breast quadrant, and diffuse - involving the entire breast (35 patients or $76.1 \%$ ). Segmental distribution, reflecting the ductal distribution, was detected in 11 patients (23.9\%). Thomassin-Naggara et al. emphasize that "the more extensive distribution of enhancement is, the less suspicious for malignancy it is". 8 Agrawal et al. showed that the diffuse enhancement was highly suggestive of benign lesions. ${ }^{24} \mathrm{On}$ the other hand, segmental NME was considered the most suspicious, with the positive predictive value (PPV) for malignancy from $76 \%$ to $100 \%$, confirmed with the study by Tozaki and Fukuda. ${ }^{25}$ Our results show that FCCs mainly show the more extensive distribution - regional and diffuse, suggesting benign aetiology of the condition.

The internal pattern of enhancement in our study was heterogeneous and clumped in 36 cases $(78.3 \%)$. The stippled enhancement was noted in eight patients $(17.4 \%)$ and homogeneous in two patients $(4.3 \%)$. According to the previous studies (Thomassin-Naggara et al., Tozaki and Fukuda), when the enhancement is heterogeneous or clumped the risk of malignancy is considered high, with the PPV of $53-58 \%$, while the homogenous enhancement has low PPV for malignancy, up to $5 \%$. The stippled enhancement represents the normal breast parenchymal enhancement. ${ }^{8,25}$ Since almost $80 \%$ of the cases in our study showed heterogeneous or clumped enhancement on DCE-MRI, the internal pattern can be one of the most important reasons for mimicry of the breast cancer by FCCs.

Kinetic curve enhancement reflects functional aspects of blood vessel permeability in normal and pathological breast tissue after intravenous gadolinium contrast application. According to the published data, the permanent enhancement (type 1 time-intensity curve) is seen in benign lesions in $85 \%$ of cases, the plateau (type 2) curve is seen in $36 \%$ of malignant cases and the washout (type 3 ) curve in $57 \%$ of malignant cases. ${ }^{26}$ In our study the majority of patients have type 2 curve ( 25 or $54.3 \%$ ), followed by type 1 curve in 17 patients $(37 \%)$ and type 3 in four patients (8.7\%). Generally, the published data about the kinetic curve enhancement of NME significantly differ. In the study of FCCs by Chen et al. $90 \%$ of NME lesions had the type 1 curve. ${ }^{19}$ Bartella et al. reported predominantly the type 2 curve in cases of malignant NME. ${ }^{27}$ Goto et al. did not find any differences in the kinetic curve enhancement between benign and malignant NME lesions. ${ }^{28}$ These differences may be partly due to the fact that the time-intensity curves are based on the semiquantitative analysis of gadolinium contrast uptake with the free-hand selected region of interest (ROI) on the heterogeneous areas of NME. Some recent studies suggested that the quantitative analysis using automated computer-aided diagnosis (CAD) can overcome subjectivity of the free-hand selection of ROI. ${ }^{29}$ The postcontrast enhancement rate types in our study were slow in 15 patients $(32.6 \%)$, moderate in 14 patients $(30.4 \%)$ and fast in 17 patients (37.0\%). Based on the published data, slow wash-in indicates benign lesions, medium wash-in may indicate lesions like mastitis, fresh scar, FCCs or DCIS, while the fast initial enhancement is suggestive of malignancy, especially when combined with the type 2 or the type 3 enhancement curve. ${ }^{30}$

NME lesions in our study of FCCs were associated with microcysts on T2W images in $54.3 \%$ of cases, which was suggestive of benign conditions. ${ }^{8}$

Additionally, we investigated DCE-MRI morphokinetic features of three distinct histological types of FCCs: nonproliferative lesions (11 cases or $24 \%$ ), proliferative lesions without atypia (29 cases or $63 \%$ ) and proliferative lesions with atypia (6 cases or $13 \%$ ). Our results show that nonproliferative lesions, proliferative lesions without atypia and proliferative lesions with atypia have similar morphokinetic features (distribution of NME, internal pattern of enhancement, type of TICs and wash-in) with the exception of the significant association of microcysts with proliferative hyperplasia without atypia. To our knowledge, our study is the first, which analyses the morphokinetic features of distinct histological types of FCCs on MRI examination. Our study was prompted by the report from Hartman et al. published in 2015, which anticipated a potentially important role of DCE-MRI in case of atypical hyperplasia. ${ }^{31}$ Hartman et al. showed that in women with atypical hyperplasia a lifetime risk of breast cancer approaches $30 \%$ at 25 years. This high cumulative incidence is not widely recognized, and thus screening DCE-MRI is not routinely recommended for these patients. ${ }^{13,32}$ Hartman et al. suggested more intensive screening of women with atypical hyperplasia, with DCE-MRI added to mammography, as well as the use of selective oestrogen-receptor modulators and aromatase inhibitors to prevent breast cancer in women with atypical hyperplasia. We hypothesized that the differentiation of atypical hyperplasia by DCE-MRI 
examination from other types of FCCs could be helpful in DCE-MRI screening. Our study was limited by the low number of proliferative lesions with atypia, thus necessitating further investigation.

Our study has some limitations. We did not analyse incidental findings of FCCs on DCE-MRI. Our analysis was conducted in the selected group of symptomatic patients: the lesions were suspicious of malignancy on clinical examination, mammography or ultrasonography and larger than $1 \mathrm{~cm}$ in DCE-MRI examination in $93.5 \%$ of cases. These facts may have influenced the interpretation and results of DCE-MRI examination.

In conclusion, the profile of FCCs presented as NME in DCE-MRI examination predominantly includes: unilateral regional or diffuse distribution (76.1\% of cases), heterogeneous or clumped internal pattern of enhancement (78.3\% of cases), plateau (type 2$)$ time-intensity curve (54.3\% of cases) with moderate or fast wash-in ( $67.4 \%$ of cases), and associated clustered microcysts (54.3\% of cases). Although these findings do not have the classic appearance of malignancy, they are sufficiently suspicious to recommend the biopsy, as final BIRADS 4 category. In case of NME, proliferative lesions without atypia are the most frequent type of FCCs $(63 \%)$, followed by nonproliferative lesions $(24 \%)$, and proliferative lesions with atypia (13\%). DCE-MRI cannot show the subtle histological differences between nonproliferative lesions, proliferative lesions without atypia and proliferative lesions with atypia. According to some novel data, MR diffusion-weighted imaging (DWI), related to tissue cellularity and thermal motion of water molecules instead of permeability of blood vessels after the contrast uptake, may be more specific than DCE-MRI to define the benign nature of FCCs. ${ }^{33,34}$

\section{References}

1. Guray M, Sahin AA. Benign breast diseases: classification, diagnosis, and management. Oncologist 2006; 11: 435-49. doi: 10.1634/theoncologist.11-5-435

2. Dupont WD, Page DL. Risk factors for breast cancer in women with proliferative breast disease. N Engl J Med 1985; 312: 146-51. doi: 10.1056/ NEJM198501173120303

3. Dupont WD, Parl FF, Hartmann WH, Brinton LA, Winfield AC, Worrell JA, et al. Breast cancer risk associated with proliferative breast disease and atypical hyperplasia. Cancer 1993; 71: 1258-65.

4. Ellis IO. Intraductal proliferative lesions of the breast: morphology, associated risk and molecular biology. Modern Pathology 2010; 23(Suppl 2): S1-7. doi: 10.1038/modpathol.2010.56

5. O'Flynn EAM. Diagnosis of primary breast cancer. In: Luna A, Vilanova JC, Celso Hygino Da Cruz Jr. LC, Rossi SE, editors. Functional imaging in oncology: clinical applications. Berlin Heidelberg: Springer-Verlag; 2014. p. 816-37.
6. D'Orsi CJ, Sickles EA, Mendelson EB, Morris EA. ACR BI-RADS atlas, breast imaging reporting and data system. Reston, VA: American College of Radiology; 2013.

7. Millet I, Pages E, Hoa D, Merigeaud S, Curros Doyon F, Prat X, et al. Pearls and pitfalls in breast MRI. Br J Radiol 2012; 85: 197-207. doi: 10.1259/ bjr/47213729

8. Thomassin-Naggara I, Salem C, Darai E, Bazot M, Uzan S, Marsault C, et al. Non-masslike enhancement on breast MRI: interpretation pearls. J Radiol 2009; 90: 269-75. doi: JR-04-2009-90-4-0221-0363-101019-200904045

9. Yabuuchi H, Matsuo $\mathrm{Y}$, Kamitani $\mathrm{T}$, Setoguchi, Okafuji $\mathrm{T}$, Soeda $\mathrm{H}$, et al. Non-mass-like enhancement on contrast-enhanced breast MR imaging: lesion characterization using combination of dynamic contrast-enhanced and diffusion-weighted MR images. Eur J Radiol 2010; 75: 126-32. doi: 10.1016/j.ejrad.2009.09.013

10. Nadrljanski M, Markovic BB, Milosevic ZC. Breast ductal carcinoma in situ (DCIS): morphologic and kinetic MRI findings. Iran J Radiol 2013; 10: 99-102. doi: 10.5812/iranjradiol.4876

11. Nadrljanski M, Milošević ZČ, Plešinac-Karapandžić V, Goldner B. Značaj magnetne rezonancije dojki u dijagnostici duktalnog karcinoma in situ. Srp Arh Celok Lek 2013; 141: 402-8. doi: 10.2298/SARH1306402N

12. Japan Association of Breast and Thyroid Sonology. Guideline for breast ultrasound: management and diagnosis. Tokyo: Nankodo Co.; 2004. p. 35-7.

13. Sardanelli F, Boetes C, Borisch B, Decker T, Federico M, Gilbert FJ, et al. Magnetic resonance imaging of the breast: Recommendations from the EUSOMA working group. Eur J Cancer 2010; 46: 1296-316. doi: 10.1016/j. ejca.2010.02.015

14. Nadrljanski MM, Miloševic ZC, Plešinac-Karapandžic V, Maksimovic R. MRI in the evaluation of breast cancer patient response to neoadjuvant chemotherapy: predictive factors for breast conservative surgery. Diagn Interv Radiol 2013; 19: 463-70. doi: 10.5152/dir.2013.13201

15. Povoski SP, Neff RL, Mojzisik CM, O'Malley DM, Hinkle GH, Hall NC, et al. A comprehensive overview of radioguided surgery using gamma detection probe technology. World J Surg Oncol 2009; 7: 7-11. doi: 10.1186/14777819-7-11

16. Santen RJ, Mansel R. Benign Breast Disorders. N Engl J Med 2005; 353: 275 85. doi: 10.1056/NEJMra035692

17. Goehring C, Morabia A. Epidemiology of benign breast disease, with special attention to histologic types. Epidemiol Rev 1997; 19: 310-27.

18. Wu C, Ray RM, Lin MG, Gao DL, Horner NK, Nelson ZC, et al. A case-control study of risk factors for fibrocystic breast conditions. Am J Epidemiol 2004; 160: 945-60. doi: 10.1093/aje/kwh318

19. Chen JH, Liu H, Baek HM, Nalcioglu O, Su MY. MR imaging features of fibrocystic change of the breast. Magn Reson Imaging 2008; 26: 1207-14. doi: 10.1016/j.mri.2008.02.004

20. Chen JH, Nalcioglu O, Su MY. Fibrocystic change of the breast presenting as a focal lesion mimicking breast cancer in MR imaging. J Magn Reson Imaging 2008; 28: 1499-505. doi: 10.1002/jmri.21455

21. van den Bosch MA, Daniel BL, Mariano MN, Nowels KN, Birdwell RL, Fong $\mathrm{KJ}$, et al. Magnetic resonance imaging characteristics of fibrocystic change of the breast. Invest Radiol 2005; 40: 436-41.

22. Kiyak $G$, Asik E, Yazgan A. Magnetic resonance imaging characteristics of fibrocystic change of the breast. Bratisl Lek Listy 2011; 112: 506-9.

23. Jansen SA, Fan X, Karczmar GS, Abe H, Schmidt RA, Giger M, et al. DCE MRI of breast lesions: Is kinetic analysis equally effective for both: mass and nonmass-like enhancement? Med Phys 2008; 35: 3102-9. doi: $10.1118 / 1.2936220$

24. Agrawal G, Su MY, Nalcioglu O, Feig SA, Chen JH. Significance of breast lesion descriptors in the ACR BIRADS MRI lexicon. Cancer 2009; 115: 1363-80. doi: $10.1002 /$ cncr.24156

25. Tozaki M, Fukuda K. High-spatial-resolution MRI of non-masslike breast lesions: interpretation model based on BI-RADS MRI descriptors. AJR Am J Roentgenol 2006; 187: 330-7. doi: 10.2214/ajr.187.3.w330

26. Rahbar $\mathrm{H}$, Partridge SC. Multiparametric MR imaging of breast cancer. Magn Reson Imaging Clin N Am 2016; 24: 223-38. doi: 10.1016/j. mric.2015.08.012 
27. Bartella L, Liberman L, Morris EA, Dershaw DD. Nonpalpable mammographically occult invasive breast cancers detected by MRI. AJR Am J Roentgenol 2006; 186: 865-70. doi: 10.2214/AJR.04.1777

28. Goto M, Ito H, Akazawa K, Kubota T, Kizu O, Yamada K, et al. Diagnosis of breast tumors by contrast-enhanced MR imaging: comparison between the diagnostic performance of dynamic enhancement patterns and morphologic features. J Magn Reson Imaging 2007; 25: 104-12. doi: 10.1002/ jmri.20812

29. Newell D, Nie K, Chen JH, Hsu CC, Yu HJ, Nalcioglu O, et al. Selection of diagnostic features on breast MRI to differentiate between malignant and benign lesions using computer-aided diagnosis: differences in lesions presenting as mass and non-mass-like enhancement. Eur Radiol 2010; 20: 771-81. doi: 10.1007/s00330-009-1616-y

30. Kaiser WA. Signs in MR-Mammography. Berlin Heidelberg: Springer-Verlag 2008

31. Hartmann LC, Degnim AC, Santen RJ, Dupont WD, Ghosh K. Atypical hyperplasia of the breast - risk assessment and management options. $N$ Engl Med 2015; 372: 78-9. doi: 10.1056/NEJMsr1407164

32. Saslow D, Boetes C, Burke W, Harms S, Leach MO, Lehman CD, et al. American Cancer Society guidelines for breast screening with MRI as an adjunct to mammography. CA Cancer J Clin 2007; 57: 75-89.

33. Ochi M, Kuroiwa T, Sunami S, Murakami J, Murakami J, Miyahara S, Nagaie T, et al. Diffusion-weighted imaging (b value $=1500 \mathrm{~s} / \mathrm{mm}(2)$ ) is useful to decrease false-positive breast cancer cases due to fibrocystic changes. Breast Cancer 2013; 20: 137-44. doi: 10.1007/s12282-011-0319-9

34. Nadrljanski MM, Gusic N, Milovanovic Z, Plesinac-Karapandzic V, Radulovic $\mathrm{O}$, Milosevic ZC. Fibrocystic changes of the breast: lesion characterization using dynamic contrast-enhanced and diffusion-weighted MR images. ECR 2013, EPOS. Available at: http://dx.doi.org/10.1594/ecr2013/C-0868. 